\title{
Fabrication of high aspect ratio microfiber arrays that mimic gecko foot hairs
}

\author{
LIU ShiYuan ${ }^{1,2^{*}}$, ZHANG Peng ${ }^{1}$, LÜ Hao ${ }^{1}$, ZHANG ChuanWei ${ }^{2} \&$ XIA Qi ${ }^{2}$ \\ ${ }^{1}$ Wuhan National Laboratory for Optoelectronics, Huazhong University of Science and Technology, Wuhan 430074, China; \\ ${ }^{2}$ State Key Laboratory of Digital Manufacturing Equipment and Technology, Huazhong University of Science and Technology, Wuhan 430074, \\ China
}

Received June 24, 2011; accepted September 23, 2011

\begin{abstract}
In nature, geckos have developed complex adhesion structures capable of smart adhesion, which is the ability to cling to different smooth and rough surfaces, even ceilings, and detach at will. The hierarchical structure of gecko foot hairs consists of microscale setae, branches and nanoscale spatulae, which contributes to their strong adhesion on different surfaces. In this paper, we propose a simple and low-cost method for fabricating two-level high aspect ratio microfiber arrays that mimic gecko foot hairs. SU-8 photoresist was used and single-level SU-8 microfiber arrays were obtained by a thick film photolithography process. Single-level polydimethyl-siloxane (PDMS) microfiber arrays were also obtained by a micromolding process and the master template for this process was fabricated using inductively coupled plasma (ICP) technology. Using the silicon mold with deep-hole arrays as a substrate, an SU-8 layer with microhole arrays was added to it using thick film photolithography and it formed a double stack mold from which the two-level hierarchical PDMS microfiber arrays were replicated. Water contact angle tests showed that the two-level hierarchical structures are extremely hydrophobic (about $148.5^{\circ}$ compared with the Tokay gecko's $160^{\circ}$ ).
\end{abstract}

gecko foot hairs, hierarchical structure, SU-8 photoresist, polydimethyl-siloxane (PDMS), double stack mold

Citation: Liu S Y, Zhang P, Lü H, et al. Fabrication of high aspect ratio microfiber arrays that mimic gecko foot hairs. Chin Sci Bull, 2012, 57: 404-408, doi: 10.1007/s11434-011-4836-x

The development of a dry adhesive fully based on van der Waals forces is highly desirable because of its wide potential application in the deployment and disassembly of microelectromechanical system (MEMS) devices such as wallclimbing robots, micro-manipulation and micro-assembly. Most traditional man-made adhesives with high adhesion strength do not detach easily and are not reusable because tacky surfaces are easily contaminated by adhering materials. In contrast, geckos have the ability to cling to different smooth and rough surfaces and detach at will, which is not easily achieved by traditional man-made adhesives. Such a strong adhesion ability is attributed to the hierarchical structure of gecko foot hairs, which consists of microscale setae, branches and nanoscale spatulae. Each toe of the gecko foot has about 15-20 rows of lamellae and each la-

*Corresponding author (email: shyliu@ mail.hust.edu.cn) mella has thousands of setae that are about 30-130 $\mu \mathrm{m}$ long and $4-10 \mu \mathrm{m}$ in diameter, which is one tenth that of a human hair. Each seta further branches out into hundreds of spatulae, which are about $2-5 \mu \mathrm{m}$ long and are 100-200 $\mathrm{nm}$ in diameter. The nanoscale spatulae are in intimate contact with various surfaces and they generate strong adhesion of about $10 \mathrm{~N} / \mathrm{cm}^{2}$ using van der Waals forces. Autumn et al. [1] measured the maximum adhesion force of a single seta and found it to be $194 \pm 25 \mu \mathrm{N}$ after sliding over $5 \mu \mathrm{m}$ and this is nearly ten times greater than that predicted using whole animal estimates.

The strong adhesion ability of the gecko provides inspiration for the design and fabrication of a new kind of biomimetic dry adhesive [2-4]. In recent years, many fabrication approaches have been reported for fiber arrays that mimic gecko foot hairs [5,6]. These include producing vertically aligned multiwall carbon nanotube (VA-MWNT) 
arrays [7,8], casting within nanoporous anodic aluminum oxide (AAO) membranes $[9,10]$ and producing unique silicon, photoresist or silicone rubber molds including positive and negative molds. This has been done by lithography and microfabrication methods followed by casting the final dry adhesive products [11-16]. Many of these methods are complicated, require specific expensive facilities or could not achieve satisfactory hierarchical structures for small size and high aspect ratio structures.

SU-8 is a commonly used epoxy-based negative photoresist. It is a very viscous polymer that can be spun or spread with a thickness ranging from $0.1 \mu \mathrm{m}$ to $2 \mathrm{~mm}$ and it can still be processed using the standard lithography process. The thick photoresist lithography process, also known as the UV-LIGA process, uses ultraviolet (UV) lithography to obtain high aspect ratio photoresist structures similar to the LIGA process. Recently, Greiner et al. [17] investigated the effect of pillar radius, aspect ratio and preload on the adhesion of bio-inspired micropatterned polydimethyl-siloxane (PDMS) structures. These were fabricated by soft-molding on arrays of holes made by the lithographic patterning of thick SU-8 films. Although the process using SU-8 did provide a promising means to fabricate microfiber arrays that mimic gecko foot hairs, only single-level structures were obtained according to their report. Additionally, del Campo and Greiner [18] reviewed several methods for the production of high aspect ratio structures from SU-8 films in detail. They particularly described a straightforward strategy that was based on layer-by-layer processes where multiple coating/irradiation steps were concatenated or iterated, and they all had defined structures at different levels. Applying this strategy, they succeeded in fabricating two-level structures containing base pillars with a diameter of $50 \mu \mathrm{m}$ and a height of $40 \mu \mathrm{m}$, and top pillars with a diameter of 9 $\mu \mathrm{m}$ and a height of $35 \mu \mathrm{m}$. However, because the SU-8 photoresist was used directly as the material for the twolevel structures this strategy was limited to smaller size and higher aspect ratio structures, especially for the top-level structure.

In this paper, we attempt to improve Greiner et al.'s method and del Campo and Greiner's strategy and we propose a simple and low cost method to fabricate two-level high aspect ratio microfiber arrays that mimic gecko foot hairs. Two-level hierarchical PDMS microfiber arrays were replicated using a double stack mold, which was formed from an SU-8 layer by thick film photolithography on a silicon master template by inductively coupled plasma (ICP) etching. We start by describing two fabrication methods for single-level structures using a thick film lithography process and an ICP process, respectively, as they provide a basis for the fabrication of the double stack mold and the two-level hierarchical microfiber arrays. The hydrophobic characteristic of the two-level microfiber arrays was verified by a water contact angle test.

\section{Single-level SU-8 microfiber arrays by a thick photoresist lithography process}

To fabricate SU-8 microfiber arrays, two layers of SU-8 photoresist were coated onto a silicon wafer separately. A schematic of the SU-8 microfiber arrays fabrication processes are shown in Figure 1. A thin SU-8 photoresist layer was spun onto a silicon wafer at a speed of $4000 \mathrm{r} \mathrm{min}^{-1}$ for $150 \mathrm{~s}$. The layer was baked for $5 \mathrm{~min}$ and exposed to UV light for $70 \mathrm{~s}$ followed by a post-bake for $5 \mathrm{~min}$. This crucial layer enhances the adherence between the high aspect ratio fiber and the substrate [16]. Another SU-8 layer was spun onto the first exposed SU-8 layer at a speed of 500 $\mathrm{r} \min ^{-1}$ for $15 \mathrm{~s}$ followed by $900 \mathrm{r} \mathrm{min}^{-1}$ for $100 \mathrm{~s}$. The estimated thickness of this layer was 80 to $120 \mu \mathrm{m}$, which determined the height of the fibers. The contact mask that was used for photolithography on the SU-8 photoresist is a triangular packing arrangement, which has a higher area fraction than that of a square packing arrangement with a 10 to $25 \mu \mathrm{m}$ circular clear area diameter. After the pre-bake for about $40 \mathrm{~min}$, the unexposed SU-8 photoresist was exposed to UV light for $70 \mathrm{~s}$. After the post-bake for $30 \mathrm{~min}$, the wafer was then developed for $5 \mathrm{~min}$ to fully remove the unexposed photoresist and an SU-8 microfiber array was obtained, as shown in Figure 2(a).

Fully cross-linked SU-8 has a Young's modulus of about 4-5 GPa, which is too rigid and brittle for use as a structural material to mimic gecko setae. Therefore, a mold is required to replicate the fibers using another suitable polymer. As shown in Figure 2(b), when the adhesion force between two adjacent fibers is stronger than the fiber bending force the SU-8 microfibers with a high aspect ratio are prone to collapse. This is difficult to replicate using the micromolding process. Therefore, a substitute for the SU-8 microfiber template is desirable.

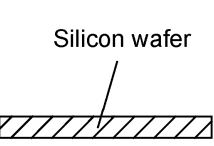

(a)

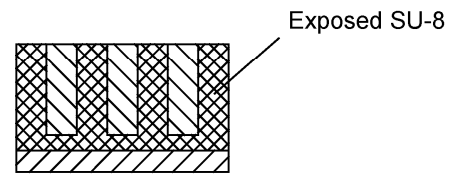

(d)

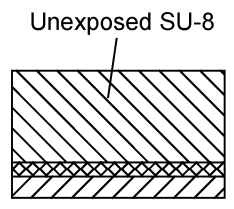

(c)

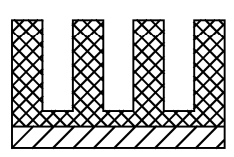

(e)
Figure 1 SU-8 microfiber fabrication processes: (a) a silicon wafer is cleaned with acetone and DI water; (b) a thin SU-8 photoresist layer is spun on the silicon wafer and is fully exposed; (c) another layer is spun on the first layer and the thickness of this layer determines the length of the fibers; (d) the second layer is exposed using the designed contact mask and (e) the SU-8 microfiber arrays are obtained by developing the exposed SU-8 photoresist. 


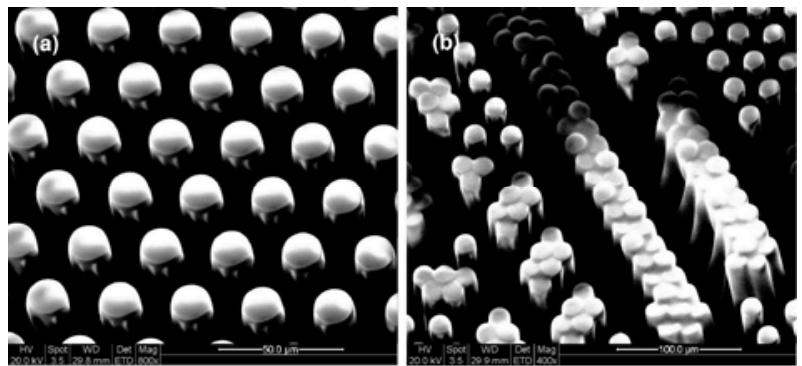

Figure 2 (a) SEM images of the vertical aligned SU-8 microfiber arrays with a $15-\mu \mathrm{m}$ diameter and an aspect ratio of 6 ; (b) collapsed SU-8 microfiber arrays with a $15-\mu \mathrm{m}$ diameter and an aspect ratio $>7$. The scale bar is $50 \mu \mathrm{m}$ for (a) and $100 \mu \mathrm{m}$ for (b).

\section{Single-level PDMS microfiber arrays by ICP and micromolding}

Instead of SU-8 photoresist we used a single-crystal silicon wafer as the master template material to fabricate the PDMS (Sylgard 184) microfiber arrays. The mask used for photolithography before ICP etching on the silicon wafer contains triangular packing arrays of circular clear areas with diameters ranging from 3 to $10 \mu \mathrm{m}$, which is similar to those in the SU-8 photolithography process. The ICP etching depth ranged from 30 to $40 \mu \mathrm{m}$. After ICP etching, acetone was used to fully remove the EPG533 photoresist from the silicon wafer. The liquid PDMS prepolymer and the curing agent were poured into a beaker and mixed thoroughly at a weight ratio of 10:1. The mixed prepolymer was then degassed for $30 \mathrm{~min}$ to remove air bubbles. The silicon master was evaporated using hexamethyl-disilazane (HMDS) as the release agent as it increases the silicon master surface activity and reduces the surface tension resulting in the cured PDMS polymer peeling off easily. The degassed PDMS prepolymer mixture was poured over the silicon master and cured at $100^{\circ} \mathrm{C}$ for $30 \mathrm{~min}$. After being fully cured the PDMS polymer was peeled from the silicon master carefully to prevent fracturing of the microfiber.

PDMS microfiber arrays with different parameters were successfully fabricated and the obtained PDMS microfiber arrays are shown in Figure 3. PDMS microfibers with an
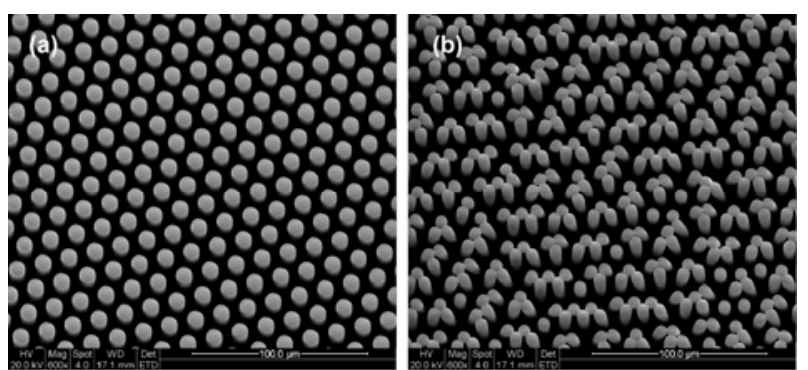

Figure 3 (a) SEM images of vertically aligned PDMS microfiber arrays with a diameter of $8 \mu \mathrm{m}$ and an aspect ratio of 5; (b) bunched PDMS microfiber arrays with a diameter of $6 \mu \mathrm{m}$ and an aspect ratio of 6 . The scale bar is $100 \mu \mathrm{m}$ for both (a) and (b). aspect ratio of 6 tend to be bunched together because of their low Young's modulus (1.7 MPa) [19].

\section{Two-level PDMS microfiber arrays by a combined SU-8 and silicon template}

In nature, each gecko pad consists of a complex hierarchical structure. This special topography allows the gecko to maintain intimate contact with almost any surface and to generate strong adhesion forces $[1,20]$. Based on the beneficial effect of such a hierarchy we fabricated two-level hierarchical microfiber arrays using a combined SU-8 and silicon template.

Figure 4 shows the schematic processes used for the fabrication. First, a silicon master template was fabricated using ICP etching, which is the same as the etching used in micromolding processes for single-level PDMS microfibers. The silicon master has a microhole array diameter of $5 \mu \mathrm{m}$. An SU-8 photoresist layer is spun on the silicon master at $500 \mathrm{r} \mathrm{min}^{-1}$ for $15 \mathrm{~s}$ followed by $2000 \mathrm{r} \mathrm{min}^{-1}$ for $100 \mathrm{~s}$. The estimated thickness of this layer is about $40 \mu \mathrm{m}$, and this determines the height of the base-level pillar. After prebaking for $40 \mathrm{~min}$ this unexposed SU-8 layer was exposed to UV light for $45 \mathrm{~s}$ and post-baked for $30 \mathrm{~min}$ until fully cured. The contact mask used for this photolithography process contains triangular packing arrays of dot arrays with a diameter of $25 \mu \mathrm{m}$. Because of the high aspect ratio feature the development process takes longer (about $10 \mathrm{~min}$ ).

Using this double layer mold, the two-level hierarchical PDMS microfiber arrays were replicated by micromolding processes, as shown in Figure 5. It is interesting to note that the hierarchical structures we obtained are smaller and have a higher aspect ratio by comparison with those reported by Greiner et al. [15]. This is due to the fact that they produced top-level microfibers directly from SU-8 photoresist through masked irradiation but we fabricated the toplevel

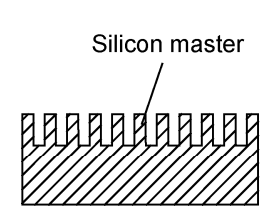

(a) ICP etching

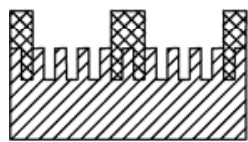

(d) Developing

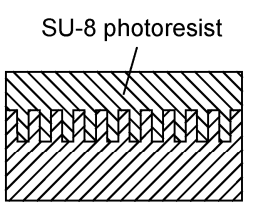

(b) Spin coating

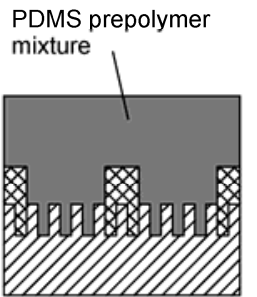

(e) Micromolding

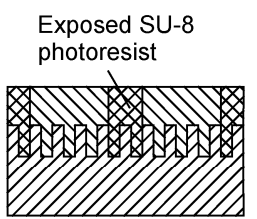

(c) Photolithography

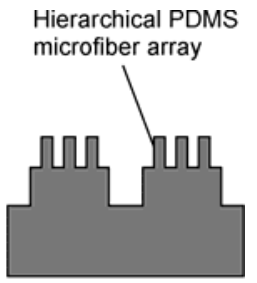

(f) Demolding
Figure 4 Schematic processes for two-level hierarchical PDMS microfiber fabrication. 

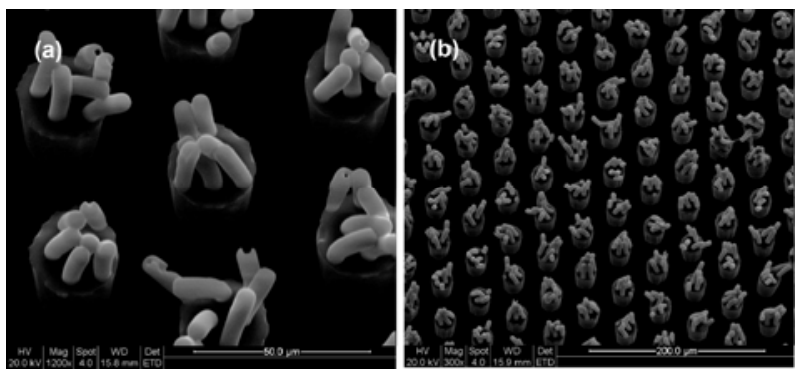

Figure 5 SEM images of two-level hierarchical PDMS microfiber arrays. The base-level pillars have a diameter of $25 \mu \mathrm{m}$ and a height of $40 \mu \mathrm{m}$, and the top-level pillars have a diameter of $5 \mu \mathrm{m}$ and a height of $35 \mu \mathrm{m}$. The scale bar is $50 \mu \mathrm{m}$ for (a) and $200 \mu \mathrm{m}$ for (b).

microfibers from PDMS by replication from a silicon template that was formed using ICP technology. However, as with the single-level PDMS microfiber arrays, the top-level PDMS microfibers also tend to collapse because of their low Young's modulus and high aspect ratio.

\section{Self-cleaning and adhesion properties}

The hydrophobic property of a surface can be obtained by increasing the surface roughness. With the help of water droplets the hydrophobic surface exhibits a self-cleaning property, which is known as the lotus effect. However, gecko pads can self-clean in dry conditions because the particles are energetically and favorably deposited on the substrate rather than remaining adhered to the gecko pads [21]. It is difficult for a synthetic adhesive to self-clean under dry conditions. Therefore, using water to clean synthetic surfaces is commonly implemented. The hydrophobic properties of both single-level and two-level PDMS microfiber arrays were verified by a water contact angle test. The observed average static water contact angle of the nonpatterned PDMS surface was found to be $102.7^{\circ}$ while those for the PDMS microfiber arrays with various fiber diameters are in the range of $131.7^{\circ}$ to $148.5^{\circ}$, as shown in Table 1 and compared with the Tokay gecko's $160^{\circ}$ [1]. The surface of the two-level hierarchical PDMS microfiber arrays exhibits a stronger hydrophobic property than that of singlelevel PDMS microfiber arrays.

Table 1 The observed average water contact angle on the surfaces of the different structures

\begin{tabular}{lcc}
\hline \multicolumn{1}{c}{ Different structures } & Diameter $(\mu \mathrm{m})$ & Contact angle $\left(^{\circ}\right)$ \\
\hline Non-patterned PDMS surface & & 102.7 \\
Single-level array & 5 & 141.6 \\
& 6 & 137.1 \\
& 7 & 136.6 \\
Two-level array & 8 & 131.7 \\
Tokay gecko & 5 & 148.5 \\
\hline
\end{tabular}

On superhydrophobic surfaces such as lotus leaves water droplets tend to slide easily because of small energy barriers [22]. However, a water droplet did not slide down on the two-level PDMS microfiber surface even when the surface was tilted vertically, as shown in Figure 6(a). As for the larger water droplet ( $>6 \mathrm{mg}$ ), the gravity of the water droplet overcame the tangential adhesion force and it slid down. However, a large water droplet $(8 \mathrm{mg}$ ) did not drop down when the two-level PDMS microfiber surface was turned over, as shown in Figure 6(b), which shows that the perpendicular adhesion force is stronger than the tangential adhesion force. The adhesion force between water and the hairy PDMS surface can be attributed to van der Waals' forces [23]. The hairy structures can make intimate contact with the water droplet and generate sufficient van der Waals' forces to hold a small droplet even when the hairy surface is hydrophobic. It can be estimated that when in contact with the rough surface the hierarchical structures can increase the contact area compared with single-level structures and nonpatterned structures.

\section{Conclusions}

In summary, we present a simple and low cost method to fabricate two-level high aspect ratio microfiber arrays that mimic gecko foot hairs by combining thick film photolithography, ICP etching and micromolding processes. SU-8 microfibers are prone to bunch together when the aspect ratio is larger than 7, which makes SU-8 difficult to use as the master template for replicating processes. Using an ICP etched silicon master template PDMS microfiber arrays were fabricated and the two-level hierarchical PDMS microfiber arrays were replicated from the double layer mold. Because of the low Young's modulus (1.7 MPa), top-level microfibers of $5 \mu \mathrm{m}$ in diameter and an aspect ratio of 7 collapsed. From the preliminary water contact angle test, we found that the synthetic surfaces of the PDMS microfiber arrays have a hydrophobic property $\left(>131.7^{\circ}\right)$ without any chemical treatment and the hierarchical feature further increases the hydrophobic property $\left(148.5^{\circ}\right)$. Additionally, the
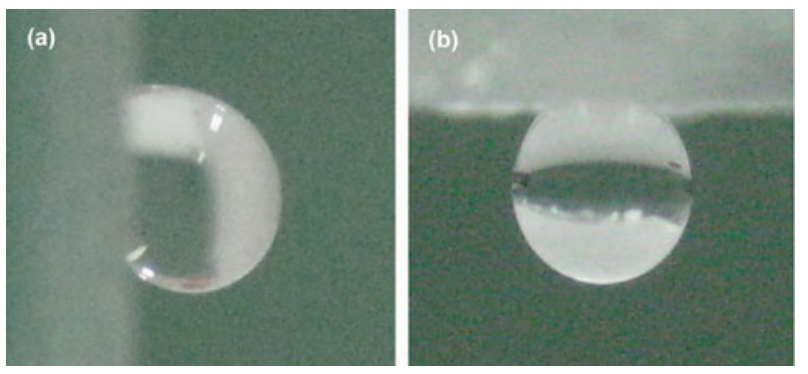

Figure 6 (a) A water droplet $(6 \mathrm{mg})$ on the two-level PDMS microfiber array film, as shown in Figure 5 with a tilt angle of $90^{\circ}$; (b) a water droplet $(8 \mathrm{mg}$ ) on the two-level PDMS microfiber array film, as shown in Figure 5 with a tilt angle of $180^{\circ}$. 
two-level PDMS surface is capable of adhering small water droplets $(8 \mathrm{mg})$ because of van der Waals' forces. In further research, the adhering ability of single-level and two-level PDMS microfiber array films with different parameters will be tested.

This work was supported by the National Natural Science Foundation of China (91023032,51005091,50775090), the Fundamental Research Funds for the Central Universities of China (2010ZD004), and the Postdoctoral Science Foundation of China (20100470052).

1 Autumn K, Liang Y A, Hsieh S T, et al. Adhesive force of a single gecko foot-hair. Nature, 2000, 405: 681-685

2 Spolenak R, Gorb S, Arzt E. Adhesion design maps for bio-inspired attachment systems. Acta Biomater, 2005, 1: 5-13

3 Yao H M, Gao H J. Mechanics of robust and releasable adhesion in biology: Bottom-up designed hierarchical structures of gecko. J Mech Phys Solids, 2006, 54: 1120-1146

4 Liu S Y, Zhang P, Cheng X, et al. Optimal design of high-aspectratio micro/nano hierarchical structures mimicking gecko foot-hairs. Adv Sci Lett, 2011, 4: 1546-1551

5 Sameoto D, Menon C. Recent advances in the fabrication and adhesion testing of biomimetic dry adhesives. Smart Mater Struct, 2010, 19: 103001

6 Boesel L F, Greiner C, Arzt E, et al. Gecko-inspired surfaces: A path to strong and reversible dry adhesives. Adv Mater, 2010, 22: 2125-2137

7 Qu L, Dai L, Wang Z L, et al. Carbon nanotube arrays with strong shear binding-on and easy normal lifting-off. Science, 2008, 322: 238-242

8 Sethi S, Ge L, Dhinojwala A, et al. Gecko-inspired carbon nanotube-based self-cleaning adhesives. Nano Lett, 2008, 8: 822-825

9 Cho W K, Choi I S. Fabrication of hairy polymeric films inspired by geckos: Wetting and high adhesion properties. Adv Funct Mater, 2008, 18: 1089-1096

10 Kustandi T S, Samper V D, Gao H, et al. Fabrication of a gecko-like hierarchical fibril array using a bonded porous alumina template. J Micromech Microeng, 2007, 17: N75-N81

11 Murphy M P, Aksak B, Sitti M. Gecko-inspired directional and controllable adhesion. Small, 2009, 5: 170-175

12 Murphy M P, Kim S, Sitti M. Enhanced adhesion by gecko-inspired hierarchical fibrillar adhesives. ACS Appl Mater Interf, 2009, 1: 849-855

13 Kim T, Jeong H E, Suh K Y, et al. Stooped nanohairs: Geometry-controllable, unidirectional, reversible, and robust gecko-like dry adhesive. Adv Mater, 2009, 21: 2276-2281

14 Jeong $\mathrm{H}$ E, Lee J, Kim H N, et al. A nontransferring dry adhesive with hierarchical polymer nanohairs. Proc Natl Acad Sci USA, 2009, 106: 5639-5644

15 Greiner C, Arzt E, del Campo A. Hierarchical gecko-like adhesives. Adv Mater, 2009, 21: 479-482

16 Aksak B, Murphy M P, Sitti M. Adhesion of biologically inspired vertical and angled polymer microfiber arrays. Langmuir, 2007, 23: 3322-3332

17 Greiner C, del Campo A, Arzt E. Adhesion of bioinspired micropatterned surfaces: Effects of pillar radius, aspect ratio, and preload. Langmuir, 2007, 23: 3495-3502

18 del Campo A, Greiner C. SU-8: A photoresist for high-aspect-ratio and 3D submicron lithography. J Micromech Microeng, 2007, 17: R81-R95

19 Roca-Cusachs P, Rico F, Martínez E, et al. Stability of microfabricated high aspect ratio structure in poly(dimethylsiloxane). Langmuir, 2005, 21: 5542-5548

20 Kim T W, Bhushan B. Adhesion analysis of multi-level hierarchical attachment system contacting with a rough surface. J Adhes Sci Technol, 2007, 21: 1-20

21 Hansen W R, Autumn K. Evidence for self-cleaning in gecko setae. Proc Natl Acad Sci USA, 2005, 102: 385-389

22 Chen W, Fadeev A Y, McCarthy T J, et al. Ultrahydrophobic and ultralyophobic surfaces: Some comments and examples. Langmuir, 1999, 15: 3395-3399

23 Autumn K, Peattie A M. Mechanisms of adhesion in geckos. Integr Comp Biol, 2002, 42: 1081-1090

Open Access This article is distributed under the terms of the Creative Commons Attribution License which permits any use, distribution, and reproduction in any medium, provided the original author(s) and source are credited. 